\title{
Pharmacological Inhibition of Soluble Epoxide Hydrolase as a New Therapy for Alzheimer's Disease
}

\author{
Christian Griñán-Ferré ${ }^{1}$ - Sandra Codony ${ }^{2}$ - Eugènia Pujol ${ }^{2}$ - Jun Yang $^{3} \cdot$ Rosana Leiva $^{2}$. \\ Carmen Escolano $^{2}$ - Dolors Puigoriol-Illamola ${ }^{1}$ - Júlia Companys-Alemany ${ }^{1} \cdot$ Rubén Corpas $^{4,5}$. \\ Coral Sanfeliu ${ }^{4,5}$ - Belen Pérez ${ }^{6}$ - M. Isabel Loza ${ }^{7}$. José Brea ${ }^{7}$. Christophe Morisseau ${ }^{3}$. \\ Bruce D. Hammock ${ }^{3} \cdot$ Santiago Vázquez ${ }^{2} \cdot$ Mercè Pallàs $^{1} \cdot$ Carles Galdeano $^{8}$
}

Published online: 2 June 2020

(C) The American Society for Experimental NeuroTherapeutics, Inc. 2020

\begin{abstract}
The inhibition of the enzyme soluble epoxide hydrolase (sEH) has demonstrated clinical therapeutic effects in several peripheral inflammatory-related diseases, with 3 compounds in clinical trials. However, the role of this enzyme in the neuroinflammation process has been largely neglected. Herein, we disclose the pharmacological validation of sEH as a novel target for the treatment of Alzheimer's disease (AD). Evaluation of cognitive impairment and pathological hallmarks were used in 2 models of agerelated cognitive decline and AD using 3 structurally different and potent $\mathrm{SEH}$ inhibitors as chemical probes. $\mathrm{sEH}$ is upregulated in brains from $\mathrm{AD}$ patients. Our findings supported the beneficial effects of central sEH inhibition, regarding reducing cognitive impairment, neuroinflammation, tau hyperphosphorylation pathology, and the number of amyloid plaques. This study suggests that inhibition of inflammation in the brain by targeting sEH is a relevant therapeutic strategy for AD.
\end{abstract}

Key Words Soluble epoxide hydrolase $\cdot$ Inflammation $\cdot$ Tau $\cdot \beta$-amyloid $\cdot$ Target engagement $\cdot$ Druggability

Highlights

- sEH levels are increased in the AD human brain and in murine models.

- Inhibition of sEH reduces oxidative stress and inflammation in murine AD models.

- AD hallmarks in $\mathrm{AD}$ mice models are reduced after treatment with $\mathrm{sEH}$ inhibitors.

- sEH inhibitors improve cognition in AD mice models.

- $\mathrm{sEH}$ can be proposed as a new pharmacological target for AD therapy.

Electronic supplementary material The online version of this article (https://doi.org/10.1007/s13311-020-00854-1) contains supplementary material, which is available to authorized users.

Christian Griñán-Ferré

christian.grinan@ub.edu

Santiago Vázquez

svazquez@ub.edu

Mercè Pallàs

pallas@ub.edu

Carles Galdeano

cgaldeano@ub.edu

Extended author information available on the last page of the article

\section{Introduction}

Chronic inflammation is recognized as a key player in both the onset and progression of Alzheimer's disease (AD) [1-3]. Indeed, $16 \%$ of the investment in ongoing clinical trials for $\mathrm{AD}$ is related to inflammation [4]. Neuroinflammation is intimately linked to the oxidative stress associated with $\mathrm{AD}[5,6]$, controlling the interactions between the immune system and the nervous system $[7,8]$. However, several antioxidant therapies and nonsteroidal anti-inflammatory drugs have failed in clinical trials. Therefore, it is of vital importance to expand the scope towards novel targets, preferably related to several pathophysiological pathways of the disease [9].

Epoxyeicosatrienoic acids (EETs) mediate vasodilatation, reduce inflammation, attenuate oxidative stress, and block the pathological endoplasmic reticulum (ER) stress response [10, 11]. The soluble epoxide hydrolase enzyme (sEH, EC 3.3.2.10, EPHX2), widely expressed in relatively high abundance in murine and human brains $[12,13]$, converts EETs and other epoxyfatty acids (EpFA) to their corresponding dihydroxyeicosatrienoic acids (DHETs), whereby diminishing, eliminating, or altering the beneficial effects of EETs [14] (Fig. 1). 


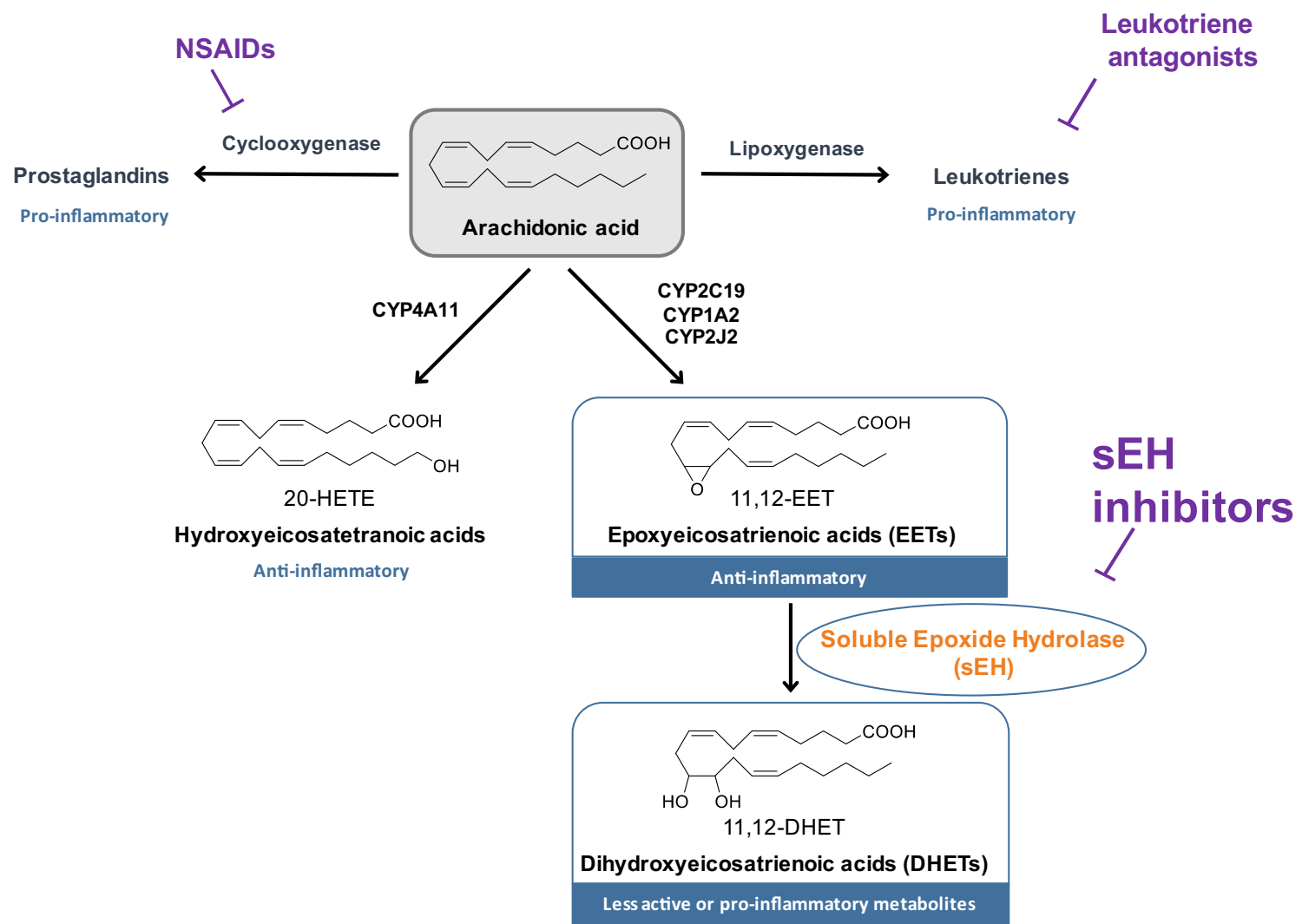

Fig. 1 The arachidonic acid cascade. The arachidonic acid (AA) cascade is a group of metabolic pathways in which AA and other polyunsaturated fatty acids are the central molecules. Metabolism via the cyclooxygenase (COX) and lipoxygenase (LOX) pathways gives rise to largely proinflammatory and proalgesic metabolites. Both pathways have been pharmaceutically targeted. CYP enzymes either hydroxylate or epoxidize AA leading to hydroxyeicosatetranoic acids (HETEs) or epoxyeicosatrienoic acids (EETs),

Considering that several lines of evidence underline a broad involvement of signaling by EETs and other EpFA in the central nervous system (CNS) function and disease $[15,16]$ and that lack of sEH by genetic deletion improves the signs of AD in a mouse model [17], we hypothesized that brain-penetrant sEH inhibitors (sEHI) would stabilize EETs in the brain, resulting in a reduction of reactive oxygen species (ROS) and diminished neuroinflammation and neurodegeneration, leading to a positive outcome in AD. To this end, we studied the neuroprotectant role mediated by sEHI in 2 models ofL AD: the senescence-accelerated mouse prone 8 (SAMP8) and 5XFAD mice models. Because SAMP8, a paradigm of late-onset AD and cognitive impairment in age, is characterized by oxidative stress, neuroinflammation, tau hyperphosphorylation, and proamilodogenic APP processing, but lacks $\beta$-amyloid $(\beta A)$ plaques [18-22], we studied plaque load and cognitive impairment in 5XFAD, a mouse model of early-onset $\mathrm{AD}$, to unveil the effect of sEHI on this AD hallmark [23, 24]. respectively. The latter compounds, which are endowed with potent antiinflammatory properties, are rapidly subjected to hydrolysis to their corresponding diols by the soluble epoxide hydrolase (sEH) enzyme. Inhibitors of $\mathrm{sEH}$ block this degradation and stabilize EET levels in vivo [14]. They also reduce the corresponding diols which have some inflammatory properties. Major CYPs that oxidize AA are listed in the figure, but many others make a contribution

\section{Methods}

Details of the experimental protocols, including chemicals, animals, novel object recognition test (NORT), biochemical and molecular methods, target engagement, drug properties characterization, and statistical analysis, are given in the Supplemental information.

\section{Results}

\section{Changes in sEH Expression in the Hippocampus from AD Patients, SAMP8, and 5XFAD}

The key question was to determine whether sEH expression differs from healthy to pathological conditions. Results demonstrated that $\mathrm{sEH}$ levels were higher in AD patients' brains (Braak III and V) in comparison with those of healthy individuals (Table S1 and Fig. 2a). Moreover, sEH expression 
was also elevated in SAMP8 and 5XFAD hippocampus in comparison with to their respective controls (Fig. 2b).

\section{On-Target Drug Inhibition of sEH}

We evaluated 3 structurally different sEH inhibitors as chemical probes [25]: TPPU $\left(\mathrm{UC1770}, \mathrm{IC}_{50}\right.$ for human $\mathrm{sEH}=$ $3.7 \mathrm{nM})$ [26], AS-2586114 $\left(\mathrm{IC}_{50}\right.$ for human $\left.\mathrm{sEH}=0.4 \mathrm{nM}\right)$ [27], and UB-EV-52 $\left(\mathrm{IC}_{50}\right.$ for human $\mathrm{sEH}=9 \mathrm{nM}$ ) [28] (Fig. 2c). Previous pharmacokinetic data suggest that TPPU, a very well characterized sEH inhibitor, can enter into the brain [29]. It is known that AS-2586114 has a prolonged action in vivo and an ability to cross the blood-brain barrier (BBB) [30,31]. UB-EV-52 is a new inhibitor somewhat related with previously reported adamantane-derived sEH inhibitors such as $t$ AUCB [32] and the clinically studied AR9281 (UC1153) [33]. To determine whether UB-EV-52 possesses drug-like characteristics, we performed in vitro ADMET assays. We found that UB-EV-52 has excellent solubility $(>100 \mu \mathrm{M}$ at $37{ }^{\circ} \mathrm{C}$ in 5\% DMSO: 95\% PBS buffer) and good microsomal stability (Table S2), and does not inhibit drug-metabolizing cytochromes or the hERG channel (Table S3). Of relevance, some cytochromes are potential off-target effects of sEH (Fig. 1), because they are situated upstream in the arachidonic acid cascade. UB-EV-52 showed less than 5\% inhibition of the studied cytochromes (CYP1A2, CYP2C9, CYP2C19, CYP3A4, and CYP2D6) at $10 \mu \mathrm{M}$ (Table S3). As a preliminary assessment of brain permeability, UB-EV-52 was subjected to the parallel artificial membrane permeation assayBBB (PAMPA-BBB), a well-established in vitro model of passive transcellular permeation [34]. UB-EV-52 was predicted to be able to cross the BBB (Table S4), which anticipates its ability to enter the brain. In order to characterize the toxicity of UB-EV-52, we evaluated cell viability in human neuroblastoma SH-SY5Y cells, using an MTT assay for cell metabolic activity and a propidium iodide stain assay for cell death. In

a

\section{b}

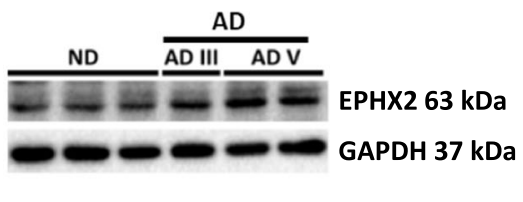

EPHX2

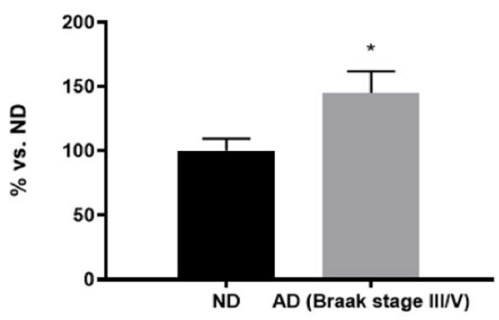

C

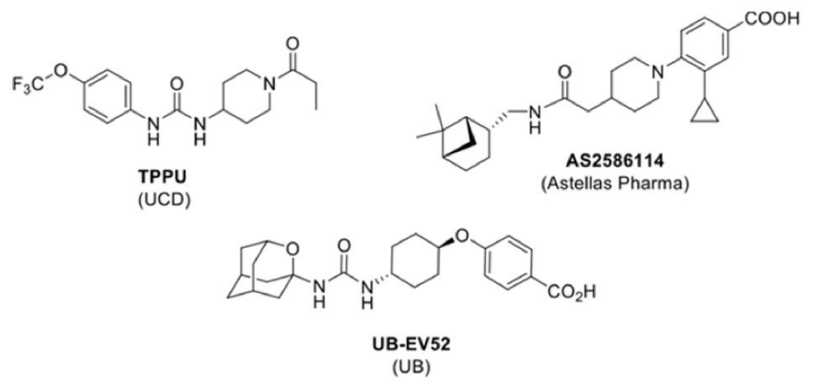

Fig. 2 Soluble epoxide inhibition and its relevance in AD. a Immunoblot of sEH (EPHX2) of human brains from AD patients (Braak stage III-V). Groups were compared by Student $t$ test $(n=4-7)$. $* p<0.05$ vs. nondemented. b Immunoblot of sEH (EPHX2) in the hippocampus of SAMP8 mice (groups were compared by Student $t$ test, $n=12-14$, $* * p<0.01$ vs. SAMR1) and 5xFAD mice (groups were compared by
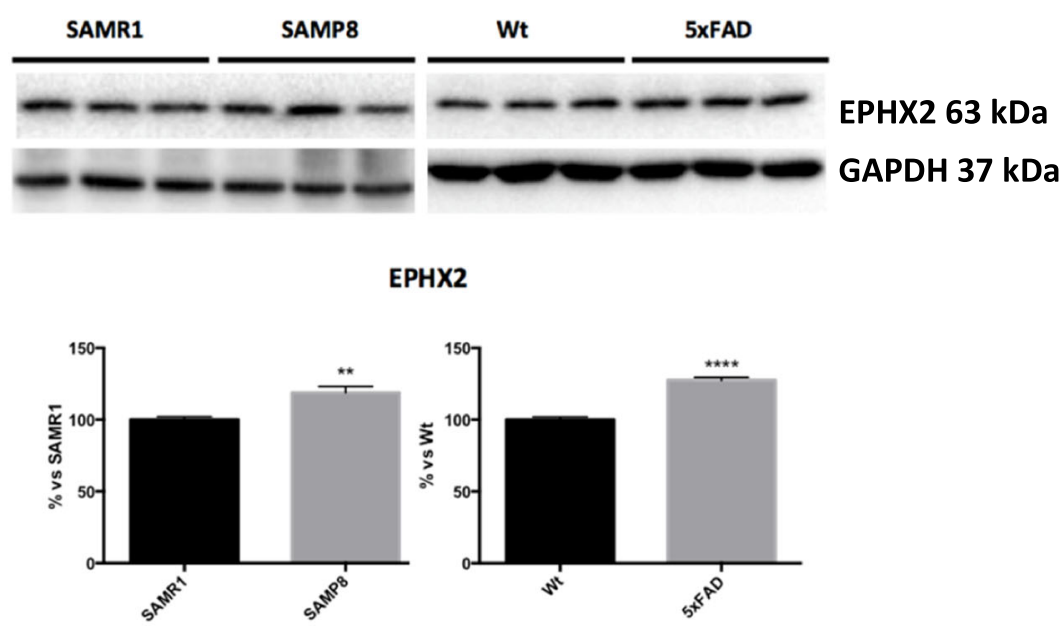

Brain

d supernatant per whole evaluation

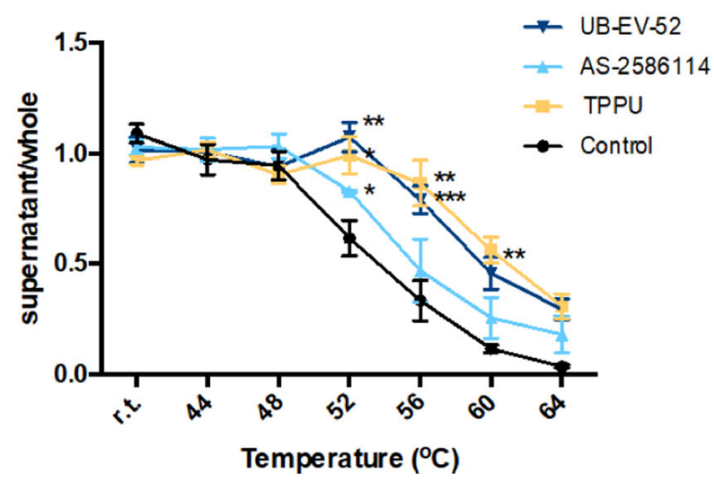

Student $t$ test, $n=12-14$, **** $p<0.0001$ vs. Wt). c Chemical structure of the sEH inhibitors employed. d CETSA experiments to monitor brain target engagement. Groups were compared by Student $t$ test or 2 -way ANOVA and post hoc Dunnett's, $n=3$ per group, $* p<0.05$, $* * p<0.01$, and $* * * p<0.001$ vs. control 
both assays, UB-EV-52 showed no cytotoxicity at 1, 10, 50, and $100 \mu \mathrm{M}$ (Table 1).

To evaluate whether sEH is the direct binding target of the inhibitors in brain tissue, we performed an in vivo thermal shift assay (CETSA) [35]. The results showed a significant shift in the sEH melting curve in the hippocampus of CD-1 mice orally treated with TPPU, AS-2586114, and UB-EV-52, demonstrating in vivo compound-induced target stabilization, providing also evidence of central action (Fig. 2d).

To demonstrate that the tested compounds reduced sEH activity, we measured levels of regulatory lipid mediators in the cortex of treated and control SAMP8 mice. As shown in Fig. S1, the levels of proinflammatory lipid mediators in the cortex such as PGD2 and TXB2 are higher in the control group. At the same time, the anti-inflammatory epoxy fatty acids, including those from -linoleic acid (EpODE) and other polyunsaturated fatty acids, are all higher in the treated groups, especially in the TPPU-treated group. These differences also verified the target engagement of inhibition of sEH in vivo.

Once demonstrated that the compounds tested were able to inhibit sEH at the brain level, we evaluated the pathological hallmarks and the cognitive impairment associated with $\mathrm{AD}$ in SAMP8 and 5XFAD.

\section{sEH Inhibitors Reduce Biomarkers of Inflammation, Oxidative Stress, and Endoplasmic Reticulum Stress}

Indicators of brain neuroinflammation were determined after oral treatment with TPPU $(5 \mathrm{mg} / \mathrm{kg} / \mathrm{day})$, AS-2586114 $(7.5 \mathrm{mg} / \mathrm{kg} /$ day), and UB-EV-52 (5 mg/kg/day) (Fig. 3a and Fig. S2). The 3 inhibitors reduced gene expression and brain protein levels of the proinflammatory cytokines IL-1 $\beta$ (interleukin- $\beta$ ), CCL3 (C-C motif ligand 3), and, importantly, TNF- $\alpha$ (tumor necrosis factor- $\alpha$ ) in SAMP8 (Fig. 3b) and in 5XFAD (data not shown). IL- $1 \beta$ is intimately involved in neuroinflammatory processes in the CNS [34], and its activity is thought to be closely tied to the process of memory consolidation $[34,35]$. Chemokines play a critical role in phagocytic activity. Concretely, CCL3 is expressed in astrocytes and is described as a component of the

Table 1 Cell viability in human neuroblastoma SH-SY5Y cells after $24 \mathrm{~h}$ exposure to UB-EV-52

\begin{tabular}{lll}
\hline UB-EV-52 concentration & $\begin{array}{l}\text { Metabolic activity (\%) } \\
\text { MTT } \\
(\text { mean } \pm \text { SEM)* }\end{array}$ & $\begin{array}{l}\text { Cell death }(\%) \\
\text { propidium iodide } \\
\text { (mean } \pm \text { SEM)* }\end{array}$ \\
\hline $0 \mu \mathrm{M}$ & $100.00 \pm 3.72$ & $0.00 \pm 0.78$ \\
$1 \mu \mathrm{M}$ & $96.84 \pm 5.01$ & $1.47 \pm 1.42$ \\
$10 \mu \mathrm{M}$ & $105.13 \pm 4.75$ & $0.95 \pm 1.31$ \\
$50 \mu \mathrm{M}$ & $118.26 \pm 8.09$ & $-0.59 \pm 1.34$ \\
$100 \mu \mathrm{M}$ & $120.43 \pm 7.30$ & $1.80 \pm 0.97$ \\
\hline
\end{tabular}

*Results of 3 independent experiments performed in quadruplicated wells inflammasome complex [36]. Of note, TNF- $\alpha$ not only is involved in AD-related brain neuroinflammation [37] but also contributes to amyloidogenesis via $\beta$-secretase regulation [38]. Additionally, these results suggest an involvement of the 2 main inflammasome-signaling pathways, NF-k $\beta$ and NLRP3 [39].

To investigate the influence of the $\mathrm{SEH}$ inhibitors in the oxidative stress process, we determined the concentration of hydrogen peroxide in the brain of SAMP8 mice. The 3 inhibitors significantly reduce hydrogen peroxide (Fig. $3 c)$. Moreover, determination of the brain oxidative machinery was addressed by evaluating the gene expression of Hmoxl, Aoxl, and protein levels of SOD1 (Fig. 3c). Hmoxl (antioxidant activity) [40] was significantly increased by UB-EV-52 and TPPU, but not by AS2586114 (Fig. 3c). qPCR analysis also demonstrated that treated SAMP8 mice had lower Aoxl expression (Fig. 3c). Aoxl controls the production of hydrogen peroxide, and under certain conditions, can catalyze the formation of superoxide [41]. Furthermore, SOD1 (antioxidant activity) protein levels were significantly increased in all treated groups (Fig. 3c), indicating a reinforcement of the antioxidant response after treatment with sEHI [42]. By contrast, in 5XFAD, a model with reduced oxidative stress, no significant changes were determined in oxidative stress parameters evaluated (data not shown).

It is known that the ER stress plays a role in the pathogenesis of neurodegenerative diseases, including $\mathrm{AD}$ [43], and sEHI attenuate activation of the ER stress response [10]. Therefore, we measured the levels of the ER stress sensors ATF-6 and IRE1 $\alpha$ (Fig. 3d). Especially, UB-EV-52 was able to reduce the levels of either protein. Furthermore, we evaluated XBP1, a major regulator of the unfolded protein response, which is induced by ER stress [44]. XBP1 was significantly reduced by UB-EV-52 and slightly decreased by AS-2586114, but not by TPPU (Fig. $3 d)$. Altogether, these results suggest that the inhibition of

Fig. 3 Role of sEH inhibitors in neurodegenerative biomarkers. a Scheme of experimental procedures in in vivo experiments. b Gene expression of neuroinflammatory markers $(I l-1 \beta, T n f-\alpha$, and $C c l 3)$ and protein levels of proinflammatory cytokines IL- $1 \beta$, TNF- $\alpha$, and CCL3 in the hippocampus of SAMP8 mice after treatment with sEH inhibitors. $\mathbf{c}$ Oxidative stress measured by hydrogen peroxide concentration in homogenates of the hippocampus. Representative gene expression of Hmox 1 and Aox 1 and representative Western blot and quantification of protein levels for (antioxidant enzyme) SOD1 in the hippocampus of SAMP8 mice after treatment with $\mathrm{SEH}$ inhibitors. d Representative Western blot and quantification of protein levels for ER stress markers ATF-6, IRE1 $\alpha$, and XBP1 in the hippocampus of SAMP8 mice after treatment with sEH inhibitors. Gene expression levels were determined by real-time PCR, cytokine protein levels by ELISA, and SOD1 by immunoblotting. Results are expressed as a mean \pm SEM and were significantly different from the control group. Groups were compared by Student $t$ test or by 1 -way ANOVA and post hoc Dunnett's, $n=4-6$ per group, $(* p<0.05, * * p<0.01$, $* * * p<0.001$, and $* * * * p<0.0001)$ vs. control. See partial correlations between selected variables in Fig. S2 and Table S7 
a

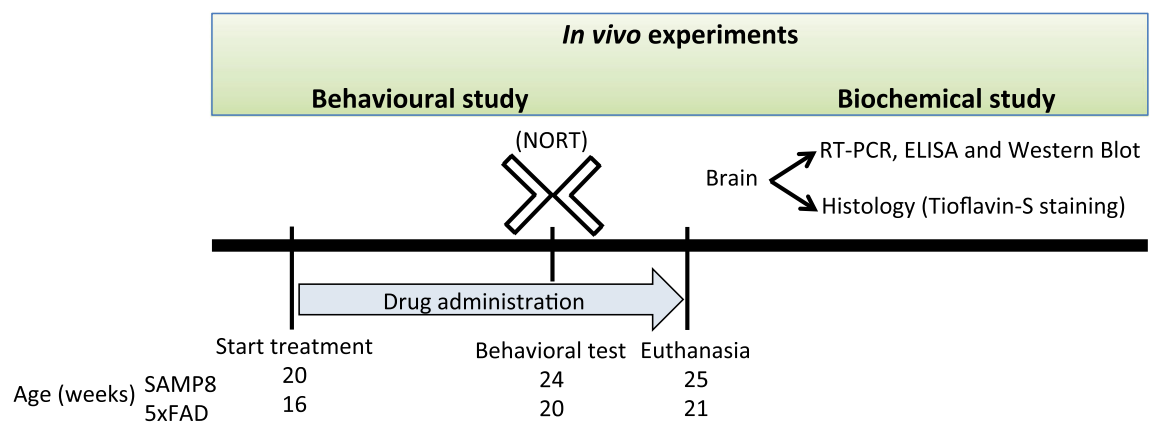

b

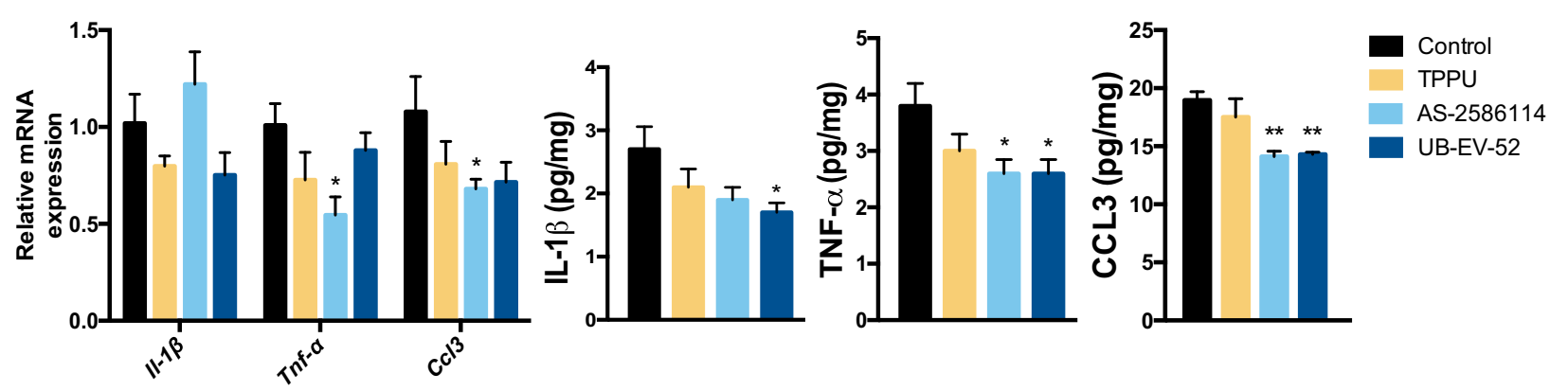

C
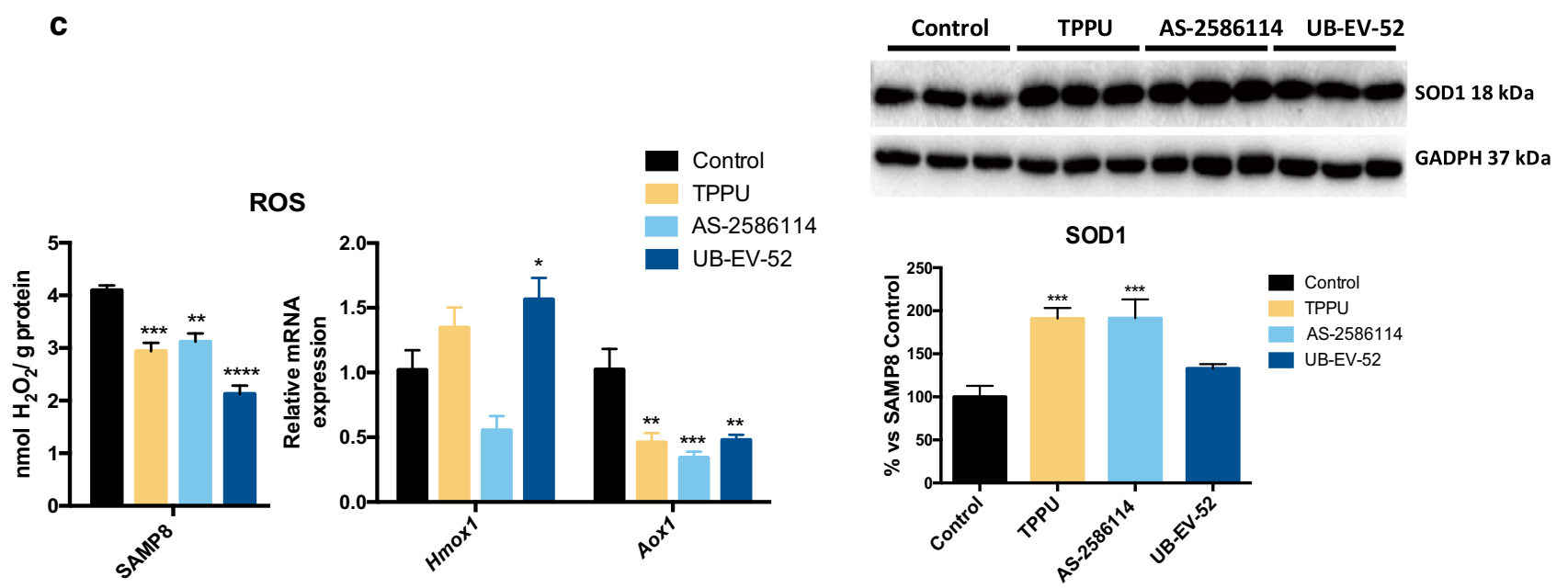

Control

TPPU

AS-2586114

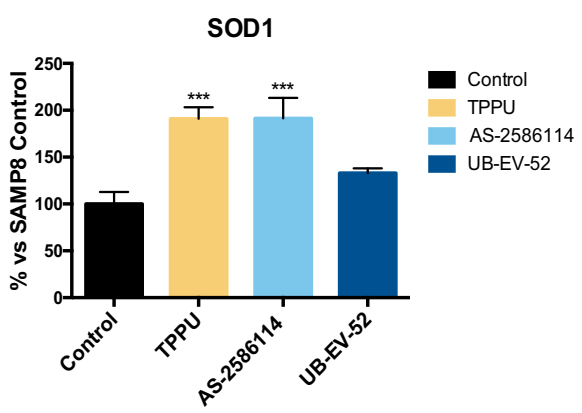

d

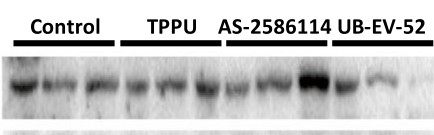

Control TPPU AS-2586114 UB-EV-52
ATF-6 $75 \mathrm{kDa}$

Control TPPU AS-2586114 UB-EV-52

-

$m-m=-m-m i \omega,+\infty=$ XBP1 29 kDa

ATF-6

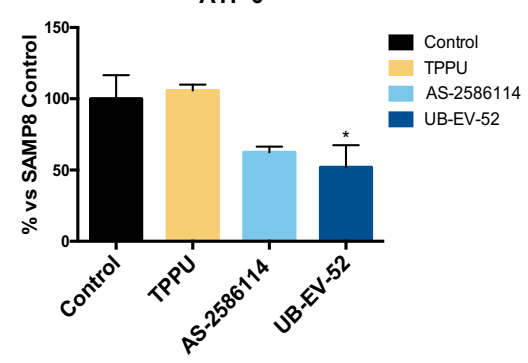

IRE1 $\alpha$

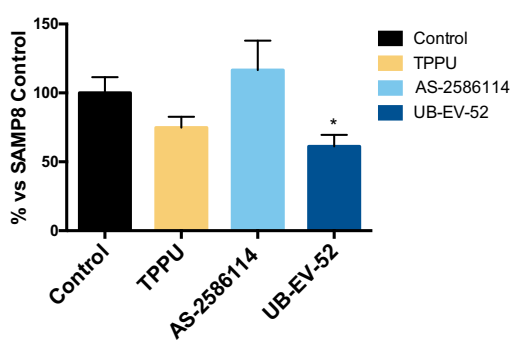

XBP1

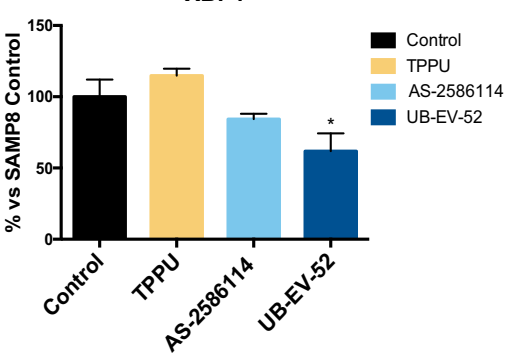


sEH protects against oxidative stress and the associated ER stress in the brain.

\section{sEH Inhibitors Modify the 2 Main Physiopathological Hallmarks of AD}

The brains of patients with AD contain 2 main physiopathological hallmarks: tangles of hyperphosphorylated tau protein and $\beta$ A plaques. As mentioned, considering that SAMP8 has disturbances in tau hyperphosphorylation and APP processing but lacks $\beta$ A plaques, 5 XFAD was used to support the protective effect of sEHI in AD hallmarks studied. On the one hand, after oral treatment of SAMP8 and 5XFAD mice with TPPU ( $5 \mathrm{mg} / \mathrm{kg} / \mathrm{day})$, AS-2586114 (7.5 mg/kg/day), and UBEV-52 (5 mg/kg/day), sEH inhibition provoked a reduction of the tau hyperphosphorylated species (Ser396 and Ser404), especially Ser404 (Fig. 4a, b), in agreement with the idea that oxidative stress can promote tau hyperphosphorylation and aggregation $[45,46]$. On the other hand, we examined the ability of the sEHI to modify the amyloid processing cascade. Whereas the 5XFAD transgenic mouse model develops early aggressive hallmarks of amyloid burden and cognitive loss [47], SAMP8 is characterized by an abnormal amyloid precursor protein (APP) processing, with a misbalance to the amyloidogenic pathway. Importantly, $C$-terminal fragment (CTF) levels are strongly implicated in neurodegeneration and the cognitive decline process in SAMP8 [21, 22]. We observed a substantial decrease in the ratio of CTFs/APP protein levels in both mice models after treatment with sEHI (Fig. $4 c, d)$. In addition, an increase of the $\operatorname{sAPP} \alpha$ and a decrease of sAPP $\beta$ supported that $\mathrm{SEHI}$ are able to shift the APP processing towards the nonamyloidogenic pathway, thereby reducing the probability of increasing $\beta A$ aggregation. Finally, treatment of 5XFAD mice with sEHI had a strong effect in reducing the number of $\beta$ A plaques stained with thioflavin-S (by an average of 40\%) (Fig. 4e), indicating the prevention of amyloid burden in a model characterized by $\beta A$ plaques formation at early ages such as 2 months.

\section{sEH Inhibitors Reduce Cognitive Impairment}

To demonstrate the efficacy on the cognitive decline of the sEH inhibitors, we performed a NORT to obtain a measure of cognition for short- and long-term memory. Treatment of both murine models with the $3 \mathrm{sEH}$ inhibitors drastically increased the discrimination index (DI). The significant increase indicates clear preservation of both memories (Fig. 5a, b). In both models, we add a comparator arm of mice treated with donepezil, which is a standard of care in the treatment of AD. As expected, donepezil treatment $(5 \mathrm{mg} / \mathrm{kg} / \mathrm{day})$ also shifted the DI to values significantly higher than zero. Remarkably, in all the conditions, the sEH inhibitors reduced cognitive decline better than donepezil.

\section{Discussion}

Our results suggest that the pharmacological stabilization of EETs in the brain has the potential to address multiple etiologies and physiopathological processes of $\mathrm{AD}$, i.e., neuroinflammation, ER stress, and oxidative stress, increasing the chances of success of future therapies based on SEH inhibition. Although the decisive role of sEH inhibition in multiple inflammation-related diseases has been studied, only a few investigations have been conducted about its crucial role in the neuroinflammation process $[15,16,30]$. Besides, the question of if neuroinflammation is the malicious driver or "just" a consequence still represents an important conundrum in the $\mathrm{AD}$ field. Our findings reinforce the idea that neuroinflammation might drive the pathogenic process in $\mathrm{AD}$. A partial correlation calculation has demonstrated that the antiinflammatory effects of sEH inhibitors correspond with changes in $\mathrm{AD}$ hallmarks, slowing the progression of the disease and pushing up the cognitive capabilities in the studied animal models (Fig. S3-S4 and Table S5-S6).

A characteristic feature of acute inflammatory processes is a general increase in the levels of classic proinflammatory eicosanoids (prostaglandins, leukotrienes, and thromboxanes). In neurodegenerative diseases, there is a basal, chronic, and silent inflammation that is more related to a disbalance in proinflammatory and anti-inflammatory cytokines, such as those studied IL-1 $\beta$ [36], CCL3 [49], and TNF- $\alpha$, as well as by acting on different mechanisms implied in neurodegeneration (e.g., increase of the oxidative stress, increase in the glutamate pathway, among others). Importantly in our landscape, $\beta A$ activates inflammasomes that, in turn, mediate IL- $1 \beta$ maturation in microglial cells [47]. This allowed us to anticipate different biological and therapeutic outcomes for sEH inhibition than for the COX and LOX pathway inhibition.

Two structurally different sEH inhibitors have been proven to be safe in human clinical trials for other peripheral indications (AR9281 for hypertension and GSK2256294 for diabetes mellitus, chronic pulmonary obstructive disease, and subarachnoid hemorrhage) $[26,50]$. A third inhibitor, EC5026, is

Fig. 4 AD hallmarks in both SAMP8 and 5xFAD mice models after treatment with $\mathrm{sEH}$ inhibitors. a and $\mathbf{b}$ Representative Western blot and quantifications for p-Tau Ser396 and p-Tau Ser404. c and d Representative Western blot and quantifications for CTFs/APP ratio, $\operatorname{sAPP} \alpha$, and $\operatorname{sAPP} \beta$. e Histological images and quantification of amyloid plaques stained with Thioflavin-S in $\mathrm{Wt}$ and $5 \mathrm{xFAD}$. Values in bar graphs are adjusted to $100 \%$ for a protein of the control group from each strain. Results are expressed as a mean \pm SEM and were significantly different from the control group. Groups were compared by Student $t$ test or by 1-way ANOVA and post hoc Dunnett's, $n=12$ per group (*significant at $p<0.05, * *$ significant at $p<0.01$, $* * *$ significant at $p<0.001$, and $* * * *$ significant at $p<0.0001)$. See partial correlations between selected variables in Fig. S2, Fig. S3, Table S7, and Table S8 
a

SAMP8

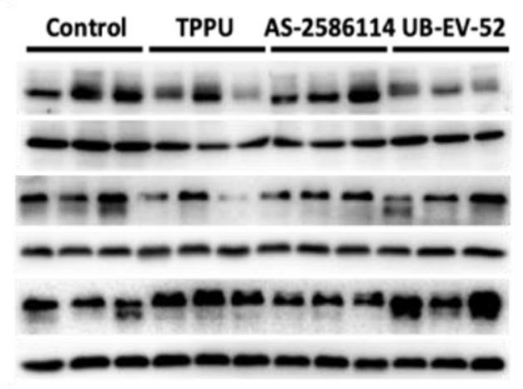

b

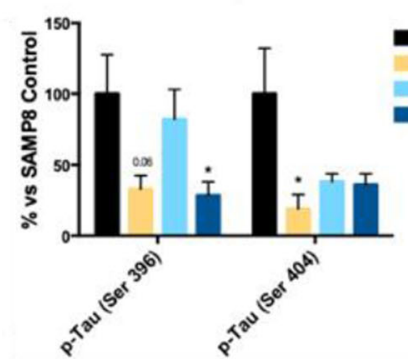

c
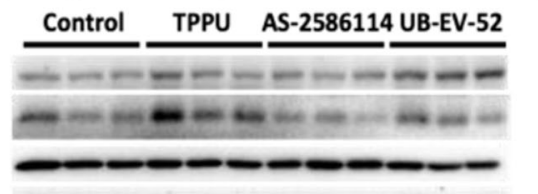

$-1---1--$

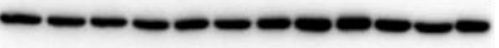
in- $-\infty-\infty-\infty-\infty-\infty$ d

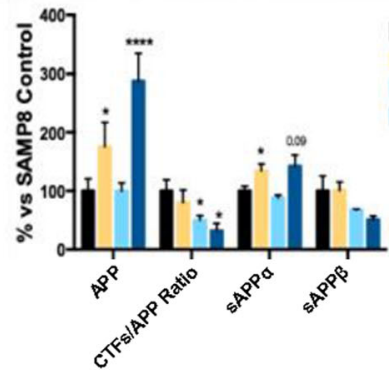

- Control

-10 TPU

-112-2586114 UB-EV-52
5XFAD
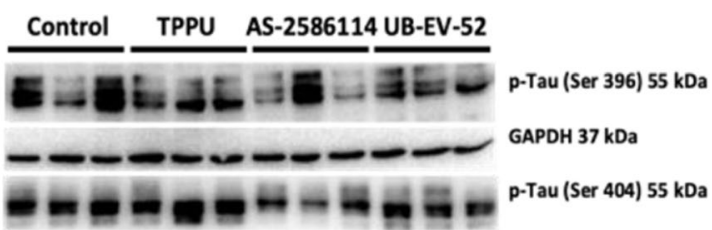

p-Tau (Ser 404) 55 kDa GAPDH $37 \mathrm{kDa}$

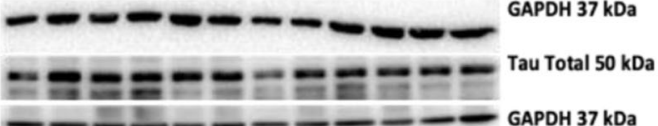

- - - - - - - GAPDH $37 \mathrm{kDa}$

Ratio p-Tau
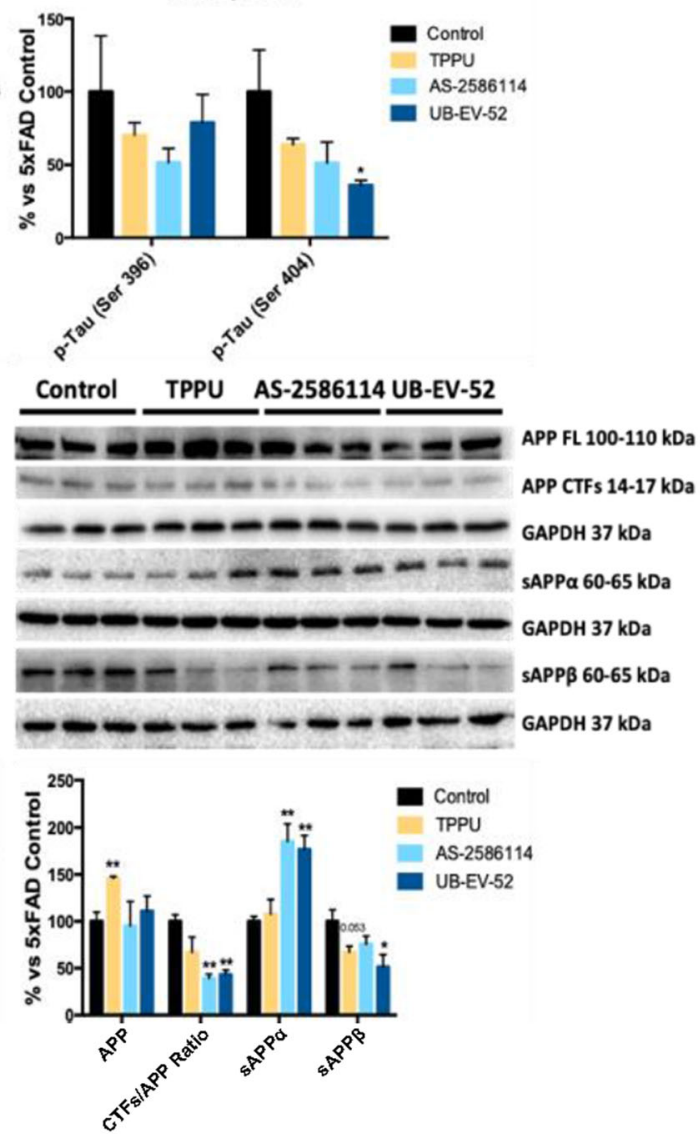

e
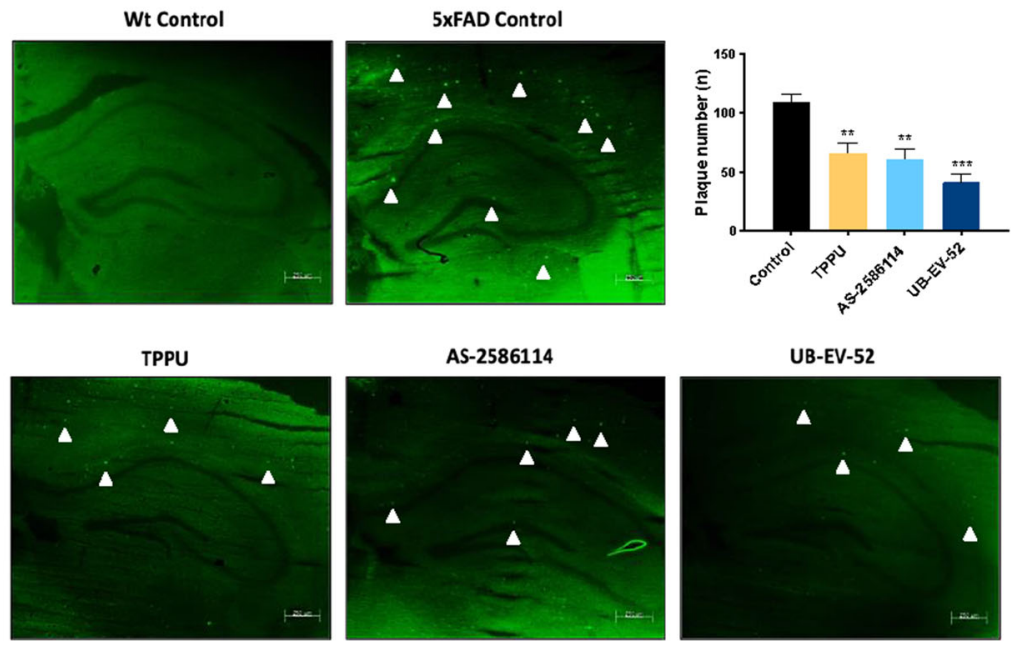


\section{a}

\section{Summary NORT}

Short-Term Memory

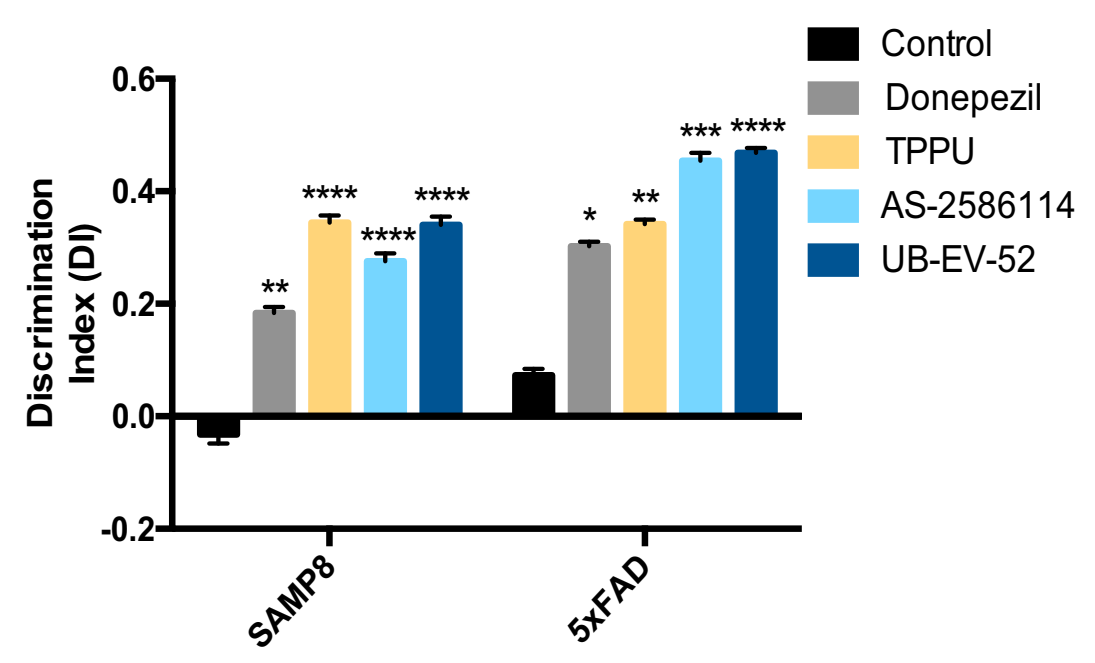

b

\section{Summary NORT Long-Term Memory}

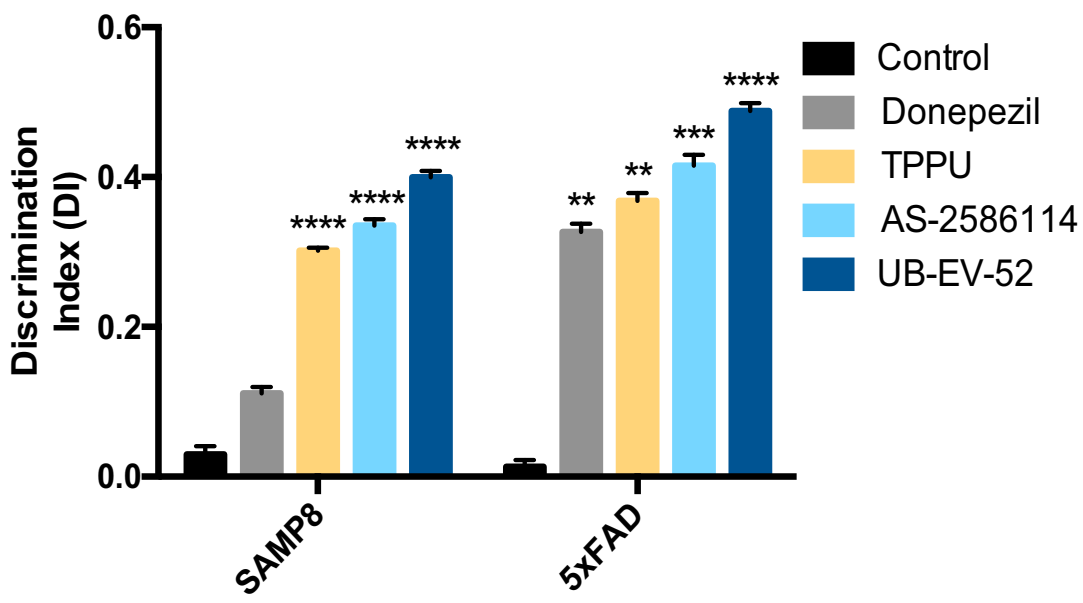

Fig. 5 Characterization of the effect of sEH inhibitors and donepezil on cognitive status in both SAMP8 and 5xFAD mice models. a Short-term memory evaluation after $2 \mathrm{~h}$ acquisition trial by discrimination index and b long-term memory evaluation after $24 \mathrm{~h}$ acquisition trial by discrimination index after exposure to novel objects. Results are expressed as a mean \pm SEM and were significantly different from the control group. Groups were compared by Student $t$ test or by 1-way ANOVA and post hoc Dunnett's, $n=12$ per group (*significant at $p<0.05, * *$ significant at $p<0.01, * * *$ significant at $p<0.001$, and $* * * *$ significant at $p<0.0001)$. See partial correlations between selected variables in Fig. S3, Fig. S4, Table S7, and Table S8

brain of $\mathrm{AD}$ patients. We have further shown that the inhibition of sEH has a plethora of beneficial central effects, such as reducing inflammation, ER stress, oxidative stress markers, ptau pathology, and the amyloid burden. Consequently, sEHI improves the functional efficacy endpoint for cognitive status in neurodegeneration and $\mathrm{AD}$ animal models.

The anti-inflammatory effect of sEHI has been demonstrated in different pathologies [16, 17, 30]. Nevertheless, considering the results obtained in this work, we cannot rule out the beneficial effects of inhibition of $\mathrm{sEH}$ by 
regulating the processes of proteostasis and OS. Those effects should be implicated in the $\beta$-amyloid plaque removal and tau hyperphosphorylation reduction. Based on the results presented in this work, we firmly believe that inhibitors of sEH could represent an entirely new standalone treatment for the treatment of AD. However, herein, we do not demonstrate, because is it beyond the scope of this study, if inhibition of sEH could also represent an add-on therapy together with more symptomatic-like drugs, i.e., donepezil.

Acknowledgments This study was supported by the Ministerio de Economía, Industria y Competitividad (Agencia Estatal de Investigación, AEI) and Fondo Europeo de Desarrollo Regional (MINECO-FEDER) (Projects SAF2017-82771, SAF2016-77703, Fundació La Caixa (project CI18-00002) and SAF2015-68749) and Generalitat de Catalunya (2017 SGR 106). S.C. and E.P. thank the Universitat de Barcelona and the Institute of Biomedicine of the Universitat de Barcelona (IBUB), respectively, for PhD grants. R.L. and D.P.-I. thank the Ministerio de Educación, Cultura y Deporte for $\mathrm{PhD}$ grants (FPU program). We would also like to thank Xunta de Galicia (ED431C 2018/21) and Ministerio de Economía, Industria y Competitividad (Innopharma Project) and Fondo Europeo de Desarrollo Regional (MINECO-FEDER). This study was also supported, in part, by grants from the National Institute of Environmental Health Sciences (NIEHS), the RIVER Award, NIEHS/R35 ES030443, and NIEHS Superfund Research Program P42 ES004699. The content is solely the responsibility of the authors and does not necessarily represent the official views of the National Institutes of Health.

Required Author Forms Disclosure forms provided by the authors are available with the online version of this article.

\section{References}

1. D. Krstic, I. Knuesel, Deciphering the mechanism underlying lateonset Alzheimer disease. Nat. Rev. Neurol. 9, 25-34 (2012).

2. V. Calsolaro, P. Edison, Neuroinflammation in Alzheimer's disease: current evidence and future directions. Alzheimer's Dementia 12, 719-732 (2016).

3. W.-Y. Fu, X. Wang, N. Y. Ip, Targeting neuroinflammation as a therapeutic strategy for Alzheimer's disease: mechanisms, drug candidates, and new opportunities. ACS Chem. Neurosci. 10, $872-879$ (2019).

4. A. T. Kodamullil, F. Zekri, M. Sood et al. Tracing investment in drug development for Alzheimer disease. Nat. Rev. Drug Discovery 16, 819 (2017).

5. E. Tönnies, E. Trushina, Oxidative stress, synaptic dysfunction, and Alzheimer's disease. J. Alzheimer's Dis. 57, 1105-1121 (2017).

6. K. J. Barnham, C. L. Masters, A. I. Bush, Neurodegenerative diseases and oxidative stress. Nat. Rev. Drug Discovery 3, 205-214 (2004).

7. M. T. Heneka, M. J. Carson, J. El Khoury et al. Neuroinflammation in Alzheimer's disease. Lancet Neurol. 14, 388-405 (2015).

8. F. L. Heppner, R. M. Ransohoff, B. Becher, Immune attack: the role of inflammation in Alzheimer disease. Nat. Rev. Neurosci. 16, 358-372 (2015).

9. S. Makin, The amyloid hypothesis on trial. Nature 559, S4-S7 (2018).
10. B. Inceoglu, A. Bettaieb, F. G. Haj, A. V. Gomes, B. D. Hammock, Modulation of mitochondrial dysfunction and endoplasmic reticulum stress are key mechanisms for the wide-ranging actions of epoxy fatty acids and soluble epoxide hydrolase inhibitors. Prostaglandins Other Lipid Mediators 133, 68-78 (2017).

11. X. Liu, C. M. Davis, N. J. Alkayed, P450 eicosanoids and reactive oxygen species interplay in brain injury and neuroprotection. Antioxid. Redox Signaling 28, 987-1007 (2018).

12. A. Marowsky, J. Burgener, J. R. Falck, J. M. Fritschy, M. Arand, Distribution of soluble and microsomal epoxide hydrolase in the mouse brain and its contribution to cerebral epoxyeicosatrienoic acid metabolism. Neuroscience 163, 646-661 (2009).

13. P. Sura, R. Sura, A. E. EnayetAllah, D. F. Grant, Distribution and expression of soluble epoxide hydrolase in human brain. J. Histochem. Cytochem. 56, 551-559 (2007).

14. C. Morisseau, B. D. Hammock, Impact of soluble epoxide hydrolase and epoxyeicosanoids on human health. Annu. Rev. Pharmacol. Toxicol. 53, 37-58 (2013).

15. L. Wang, G. Luo, L. F. Zhang, H. X. Geng, Neuroprotective effects of epoxyeicosatrienoic acids. Prostaglandins Other Lipid Mediators 138, 9-14 (2018).

16. K. M. Wagner, C. B. McReynolds, W. K. Schmidt, B. D. Hammock, Soluble epoxide hydrolase as a therapeutic target for pain, inflammatory and neurodegenerative diseases. Pharmacol. Ther. 180, 62-76 (2017).

17. H.T. Lee, K.I. Lee, C.H. Chen, T.S. Lee. Genetic deletion of soluble epoxide hydrolase delays the progression of Alzheimer's disease. J. Neuroinflammation 16, 267 (2019).

18. J. E. Morley, S. A. Farr, J. F. Flood, Antibody to amyloid $\beta$ protein alleviates impaired acquisition, retention, and memory processing in SAMP8 mice. Neurobiol. Learn. Mem. 78, 125-138 (2002).

19. T. Takeda, Senescence-accelerated mouse (SAM) with special references to neurodegeneration models, SAMP8 and SAMP10 mice. Neurochem. Res. 34, 639-659 (2009).

20. I. Akiguchi, M. Pallàs, H. Budka et al. SAMP8 mice as a neuropathological model of accelerated aging and dementia: Toshio Takeda's legacy and future directions. Neuropathology 37, 29305 (2017).

21. M. Pallàs, Senescence-accelerated mice P8: a tool to study brain aging and Alzheimer's disease in a mouse model. ISRN Cell Biol. 2012, 917167 (2012).

22. C. Griñan-Ferré, V. Palomera-Ávalos, D. Puigoriol-Illamola et al. Behaviour and cognitive changes correlated with hippocampal neuroinflammaging and neuronal markers in female SAMP8, a model of accelerated senescence. Exp. Gerontol. 80, 57-69 (2016).

23. H. Oakley, S. L. Cole, S. Logan et al. Intraneuronal $\beta$-amyloid aggregates, neurodegeneration, and neuron loss in transgenic mice with five familial Alzheimer's disease mutations: potential factors in amyloid plaque formation. J. Neurosci. 26, 10129-10140 (2006).

24. R. Kimura, M. Ohno, Impairments in remote memory stabilization precede hippocampal synaptic and cognitive failures in $5 \times \mathrm{FAD}$ Alzheimer mouse model. Neurobiol Dis. 33, 229-235 (2009).

25. C. H. Arrowsmith, J. E. Audia, C. Austin, et al. The promise and peril of chemical probes. Nat. Chem. Biol. 11, 536-541 (2015).

26. T. E. Rose, C. Morisseau, J. Y. Liu et al. 1-Aryl-3-(1-acylpiperidin4-yl)urea inhibitors of human and murine soluble epoxide hydrolase: structure-activity relationships, pharmacokinetics and reduction of inflammatory pain. J. Med. Chem. 53, 7067-7075 (2010).

27. M. Miura, I. Sato, H. Kiyohara, et al. Cyclic amino compound, or salt thereof. JP2011016743 (2011).

28. S. Vázquez, E. Valverde, R. Leiva, M. Vázquez-Carrera, S. Codony, Analogs of adamantylureas as soluble epoxide hydrolase inhibitors. WO 2017017048 (2017).

29. A. I. Ostermann, J. Herbers, I. Willenberg et al. Oral treatment of rodents with soluble epoxide hydrolase inhibitor 1-(1propanoylpiperidin-4-yl-)-3-[4-(trifluoromethoxy)phenyl]urea 
(TPPU): resulting drug levels and modulation of oxylipin pattern. Prostaglandins Other Lipid Mediators 121, 131-137 (2015).

30. N. Taguchi, S. Nakayama, M. Tanaka. Single administration of soluble epoxide hydrolase inhibitor suppresses neuroinflammation and improves neuronal damage after cardiac arrest in mice. Neurosci. Res. 111, 56-63 (2016).

31. M. Ma, Q. Ren, Y. Fujita, T. Ishima, J. C. Zhang, K. Hashimoto, Effects of AS2586114, a soluble epoxide hydrolase inhibitor, on hyperlocomotion and prepulse inhibition deficits in mice after administration of phencyclidine. Pharmacol. Biochem. Behav. 110, 98-103 (2013).

32. S. H. Hwang, H. J. Tsai, J. Y. Liu, C. Morisseau, B. D. Hammock, Orally bioavailable potent soluble epoxide hydrolase inhibitors. J. Med. Chem. 50, 3825-3840 (2007).

33. R. Chen, E. Whitcomb, V. MacIntyre, et al. Pharmacokinetics and pharmacodynamics of AR9281, an inhibitor of soluble epoxide hydrolase, in single- and multiple-dose studies in healthy human subjects. J. Clin. Pharmacol. 52, 319-328 (2013).

34. L. Di, E.H. Kerns, K. Fan, O. J. McConnell, G.T. Carter. High throughput artificial membrane permeability assay for blood-brain barrier. Eur. J. Med. Chem. 38, 223-232 (2003).

35. R. Martínez Molina, M. Jafari, M. Ignatushchenko, et al. Monitoring drug target engagement in cells and tissues using the cellular thermal shift assay. Science 341, 84-87 (2013).

36. S.S. Shaftel, S. Kyrkanides, J.A. Olschowka, J.H. Miller, R.E. Johnson, M.K. O'Banion. Sustained hippocampal IL-1 $\beta$ overexpression mediates chronic neuroinflammation and ameliorates Alzheimer plaque pathology. J. Clin. Invest. 117(6), 1595-1604 (2007).

37. H. Schneider, F. Pitossi, D. Balschun, A. Wagner, A. del Rey, H.O.Besedovsky HO. A neuromodulatory role of interleukin1 beta in the hippocampus. Proc. Natl. Acad. Sci. U. S. A. 95, 7778-83 (1998).

38. A.V. Andjelkovic, D. Kerkovich, J. Shanley, L. Pulliam, J.S. Pachter. Expression of binding sites for beta chemokines on human astrocytes. Glia. 28, 225-35 (1999).

39. M. T. Heneka, R. M. McManus, E. Latz, Inflammasome signalling in brain function and neurodegenerative disease. Nat. Rev. Neurosci. 19, 610-621 (2018).

40. Z. Si, X. Wang, Z. Zhang et al. Heme oxygenase 1 induces tau oligomer formation and synapse aberrations in hippocampal neurons. J. Alzheimers Dis. 65, 409-419 (2018).
41. R. M. Wright, L. K. Weigel, M. Varella-Garcia, G. Vaitaitis, J. E. Repine, Molecular cloning, refined chromosomal mapping and structural analysis of the human gene encoding aldehyde oxidase (AOX1), a candidate for the ALS2 gene. Redox Rep. 3, 135-144 (2016).

42. K. Murakami, N. Murata, Y. Noda, et al. SOD1 (copper/zinc superoxide dismutase) deficiency drives amyloid $\beta$ protein oligomerization and memory loss in mouse model of Alzheimer disease. J. Biol. Chem. 286, 44557-44568 (2011).

43. C. Hetz, S. Saxena, ER stress and the unfolded protein response in neurodegeneration. Nat. Rev. Neurol. 13, 477-491 (2017).

44. C. Corona, A. Pensalfini, V. Frazzini, S. L. Sensi, New therapeutic targets in Alzheimer's disease: brain deregulation of calcium and zinc. Cell Death Dis. 2, e176 (2011).

45. H. Zempel, E. Thies, E. Mandelkow, E. M. Mandelkow, Abeta oligomers cause localized $\mathrm{Ca}^{2+}$ ) elevation, missorting of endogenous Tau into dendrites, Tau phosphorylation, and destruction of microtubules and spines. J. Neurosci. 30, 11938-11950 (2010).

46. S. D. Girard, K. Baranger, C. Gauthier, M. Jacquet et al. Evidence for early cognitive impairment related to frontal cortex in the 5XFAD mouse model of Alzheimer's disease. J. Alzheimers Dis. 33, 781-796 (2013).

47. J .Couturier, I.C. Stancu, O. Schakman et al. Activation of phagocytic activity in astrocytes by reduced expression of the inflammasome component ASC and its implication in a mouse model of Alzheimer disease. J Neuroinflammation. 27, 13-20 (2016).

48. A. L. Lazaar, L. Yang, R. L. Boardley, N. S. Goyal et al. Pharmacokinetics, pharmacodynamics and adverse event profile of GSK2256294, a novel soluble epoxide hydrolase inhibitor. Br. J. Clin. Pharmacol. 81, 971-979 (2016).

49. H. Braak, E. Braak, in Cerebral Cortex, Neurodegenerative and age-related changes in structure and function of cerebral cortex, A. Peters, J. H. Morrison, Eds. (Kluwer Academic/Plenum Publisher, New York, 1999), vol. 14, pp. 475-512.

50. J Bhattacharya S, Maelicke A, Montag D. Nasal application of the galantamine pro-drug Memogain slows down plaque deposition and ameliorates behavior in 5X Familial Alzheimer's disease mice. Alzheimers Dis. 46,123-36(2015)

Publisher's Note Springer Nature remains neutral with regard to jurisdictional claims in published maps and institutional affiliations.

\section{Affiliations}

\section{Christian Griñán-Ferré ${ }^{1} \cdot$ Sandra Codony $^{2} \cdot$ Eugènia Pujol $^{2} \cdot$ Jun Yang $^{3} \cdot$ Rosana Leiva $^{2} \cdot$ Carmen Escolano $^{2}$. Dolors Puigoriol-Illamola ${ }^{1}$. Júlia Companys-Alemany ${ }^{1} \cdot$ Rubén Corpas $^{4,5} \cdot$ Coral Sanfeliu $^{4,5} \cdot$ Belen Pérez $^{6}$. M. Isabel Loza ${ }^{7}$. José Brea ${ }^{7}$. Christophe Morisseau ${ }^{3}$ - Bruce D. Hammock ${ }^{3}$ - Santiago Vázquez ${ }^{2} \cdot$ Mercè Pallàs $^{1}$. Carles Galdeano ${ }^{8}$}

1 Pharmacology Section, Department of Pharmacology, Toxicology and Medicinal Chemistry, Faculty of Pharmacy and Food Sciences, and Institut de Neurociències, University of Barcelona, Av. Joan XXIII, 27-31, E-08028 Barcelona, Spain

2 Laboratori de Química Farmacèutica (Unitat Associada al Consejo Superior de Investigaciones Científicas), Department de Farmacologia, Toxicologia i Química Farmacèutica, Facultat de Farmàcia i Ciències de de l'Alimentació y Institut de Biomedicina, Av. Joan XXIII, 27-31, E-08028 Barcelona, Spain
3 Department of Entomology and Nematology and Comprehensive Cancer Center, University of California, One Shields Avenue, Davis, CA 95616, USA

4 Institute of Biomedical Research of Barcelona, Consejo Superior de Investigaciones Científicas and Institut d'Investigacions Biomèdiques August Pi i Sunyer, Rosselló, 149, E08036 Barcelona, Spain

5 Centros de Investigacion Biomedica en red Epidemiology and Public Health, Av. Monforte de Lemos, 3-5. Pabellón 11. Planta 0, E28029 Madrid, Spain 
6 Department of Pharmacology, Therapeutic and Toxicology, Autonomous University of Barcelona, E-08193 Barcelona, Spain

7 Innopharma Screening Platform, Biofarma Research Group, Centro de Investigación en Medicina Molecular y Enfermedades Crónicas, Universidad de Santiago de Compostela, Edificio CIMUS, Av.

Barcelona, S/N, E-15706 Santiago de Compostela, Spain
8 Department of Pharmacy and Pharmaceutical Technology and Physical Chemistry, Faculty of Pharmacy and Food Sciences and Institute of Biomedicine, University of Barcelona, Av. Joan XXIII, 27-31, E-08028 Barcelona, Spain 